\title{
An Efficient TP Model Transformation Algorithm for Robust Visual Servoing in the Presence of Uncertain Data
}

\author{
Tingting Wang ${ }^{1 *}$, Yanyun $\mathrm{Bi}^{1}$, Teng $\mathrm{Hou}^{2}$, Bo Liu ${ }^{1}$, Jianfeng \\ $\mathrm{Cui}^{3}$
}

${ }^{1}$ Department of Mechanical and Electrical Engineering, Hohai University, No. 200 North Jinling Road, 213022, Changzhou City, Jiangsu Province, China, wangtt@hhuc.edu.cn, byy201809@hhu.edu.cn, liub@hhuc.edu.cn

${ }^{2}$ System Engineering Research Institute, China State Ship building Corporation, No. 1 Fengxian East Road, Haidian District, 100094, Beijing, China

${ }^{3}$ School of Electrical and Control Engineering, North University of China, No. 3, Xueyuan Road, Jiancaoping District, 030056, Taiyuan City, Shanxi Province, China, cuijf@nuc.edu.cn

* Corresponding author

\begin{abstract}
This paper presents a robust visual servoing controller based on an efficient TP model transformation method, while taking into account an uncertain image Jacobian matrix where, the camera intrinsic parameters, image features, and depth estimations are affected by unknown random uncertainties with known bounds. The convex vertex decomposition of image Jacobian matrix through uniform design greatly reduce the number of LMIs in the quasi-min-max model predictive control (MPC) scheme, in order to obtain the optimal control inputs of the constrained visual servoing system, while meeting the real-time requirements. Simulation and Experimental results demonstrate the effectiveness of the proposed method.
\end{abstract}

Keywords: TP model transformation; Uniform design; Uncertain data; quasi-min-max MPC; LMIs

\section{Introduction}

Visual servoing enables robotic systems to perform positioning or tracking tasks in a non-structural environment [1]-[2]. Traditional visual servoing can be divided into image-based visual servoing (IBVS) [3], position-based visual servoing (PBVS) [4] and hybrid visual servoing [5]. The error signal of the classical IBVS is defined in the two-dimensional image feature space directly from the camera for 
feedback to control the motion of the robot. However, this approach has some drawbacks, such as, the singularity of the image Jacobian matrix, the local minimum with large displacement, and the difficulty of dealing with constraints. Numerous advanced control schemes have been published to try to improve the control performance and conquer the drawbacks mentioned above. In order to handle singularities, [6] used Takagi-Sugeno fuzzy framework to model the IBVS. A switch controller was proposed in [7] to realize a large displacement grasping task. In [8], photometic moments are derived to improve the convergence domain. However, these methods still have not addressed the constraints explicitly which are crucial for real systems control designing. In [9], the fusion of hysteresis constraint with the image-based visual servoing manipulator system is considered. [10] is an adaptive image-based visual servoing with temporary loss of the visual signal, a homography method that uses a priori visual information is proposed to predict all of the missing feature points and to ensure the execution of IBVS. [11] proposed a path planning approach for visual servoing with elliptical projections to deal with constraints. In [12], different types of constraints are defined, and a sliding mode based approach is proposed to satisfy constraints in robot visual servoing. In addition, because of the advantage of handling constraints, several MPC-based IBVS control schemes are proposed. In [13], predictive control method for both local and global model of constrained IBVS is proposed. A quasimin-max MPC scheme is presented in [14], where the feasible solutions of LMIs depend on the vertexes of the image Jacobian matrix decomposed by the TP model transformation. In [15], TP models of the visual servoing system is reduced to improve the speed of the LMIs solution, and the algorithm is verified by experiments. However, the above mentioned methods require the knowledge of the camera intrinsic and extrinsic parameters, and the depth information should be given. Despite there are several classical calibration methods, they are time consuming, require experience, and have inherent inaccuracies. If the calibration parameters are not exactly known and accompanied with model uncertainties (such as image measurement errors and depth estimation errors, etc.), the image Jacobian matrix is difficult to estimate, thus, the visual servoing system may suffer from performance degradation and potential unpredictable response. In this paper, a robust constrained visual servoing control method in the presence of uncertain data is considered.

Many nonlinear and linear controllers could be considered to deal with a state space model with constraints [16-18]. TP model transformation method is an effective numerical method that can convert a LPV uncertain model into the canonical form of polytopic models in a unified way $[19,20]$. The implementation of the TP model transformation is a numerically tractable non-heuristic algorithm, therefore it is a useful engineering tool that can be easily executed [21, 22]. In the recent past, many control approaches and applications have been carried out on the TP model transformation [14, 23-26], including in the area of LMI-based control design, sliding model control, etc. Our past research $[14,15]$ are typical applications of TP model transformation in visual servoing area. In theory, it is 
easy to extend to the uncertain visual servoing model where the parameters of image Jacobian matrix are affected by unknown random uncertainties with known bounds. However, the computational load of TP model transformation will increase rapidly with the variable dimension of image Jacobian matrix. And the number of the convex vertexes generated by TP model transformation also directly affects the computational complexity of quasi-min-max MPC. When the uncertain parameters (e.g. the camera intrinsic parameters, image measurements, depth estimations, etc.) are considered, excessive number of LMIs may lead to conservative and impose great difficulties on the computation of feasible solutions, which brings the limitation for practical application of visual servoing control. In this paper, in order to conquer the shortcomings mentioned above, an efficient modified TP model transformation method based on the uniform design $[27,28]$ is implemented to achieve a robust visual servoing control in the presence of bounded uncertain system parameters, which satisfy the operational speed in online applications.

This article is organized as follows: Section 2 discusses the visual servoing model with uncertain parameters. The robust visual servoing controller design is presented in Section 3, which include the quasi-min-max MPC formulation for IBVS system and the efficient TP model transformation for image Jacobian matrix In Section 4, simulation and experimental results for eye-in-hand camera configuration are presented to demonstrate the effectiveness of the proposed control method. Finally, conclusions are provided in Section 5.

\section{Visual Servoing Model with Uncertain Parameters}

The aim of the visual servoing control is to minimize an error $\mathbf{e}(t)$, which is typically defined by

$$
\mathbf{e}(t)=\mathbf{s}(m(t), a)-\mathbf{s}^{*}
$$

where $\mathbf{s}(m(t), a)$ is a vector of visual features, $\mathbf{s}^{*}$ contains the desired feature values. The vector $m(t)$ is a set of image measurements to compute the visual features $\mathbf{s}(m(t), a)$, and the parameters $a$ include the camera or object model information of the visual servoing system.

Classical image-based control schemes taking the pixel coordinates of a set of image points to define the visual features, and the camera intrinsic parameters are used to make the image measurements expressed in pixels to the features. Without loss of generality, a unique camera pose can theoretically be obtained by using four stationary coplanar and non-collinear feature points denoted by $O_{\mathrm{i}}$ $\forall i=1,2,3,4$. Suppose the normalized Euclidean coordinate vectors of the feature 
points $O_{\mathrm{i}}$ expressed in the current camera coordinate frame and the desired camera coordinate frame, are defined as $\mathbf{m}_{i} \in \mathfrak{R}^{3}$ and $\mathbf{m}_{i}^{*} \in \mathfrak{R}^{3}$ with:

$$
\begin{aligned}
& \mathbf{m}_{i}=\left[\begin{array}{lll}
\frac{x_{i}}{z_{i}} & \frac{y_{i}}{z_{i}} & 1
\end{array}\right]^{T} \\
& \mathbf{m}_{i}^{*}=\left[\begin{array}{lll}
\frac{x_{i}^{*}}{z_{i}^{*}} & \frac{y_{i}^{*}}{z_{i}^{*}} & 1
\end{array}\right]^{T}
\end{aligned}
$$

For a pinhole camera model, the transformation between the pixel coordinates $\mathbf{p}_{i}=\left[\begin{array}{lll}u_{i} & v_{i} & 1\end{array}\right]^{T} \in \mathfrak{R}^{3}$ and $\mathbf{p}_{i}^{*}=\left[\begin{array}{lll}u_{i}^{*} & v_{i}^{*} & 1\end{array}\right]^{T} \in \mathfrak{R}^{3}$ of each feature point $O_{\mathrm{i}}$ can be expressed as

$$
\begin{aligned}
& \mathbf{p}_{i}=\mathbf{A} \mathbf{m}_{i} \\
& \mathbf{p}_{i}^{*}=\mathbf{A} \mathbf{m}_{i}^{*}
\end{aligned}
$$

where $\mathbf{A} \in \mathfrak{R}^{3 \times 3}$ is the upper-triangular matrix containing the camera intrinsic parameters:

$$
\mathbf{A}=\left[\begin{array}{ccc}
f_{x} & 0 & u_{0} \\
0 & f_{y} & v_{0} \\
0 & 0 & 1
\end{array}\right]
$$

including $u_{0}, v_{0} \in \mathfrak{R}$ the coordinates of the principal point, and $f_{x}, f_{y} \in \mathfrak{R}$ the product of the camera scaling factors and the focal length.

Usually, camera calibration is a costly, tedious and error prone process. It is also difficult to measure the depth online for the monocular vision. In addition, the measurement errors may be introduced by the process of image processing. In this paper, unknown random uncertainties with known bounds are considered in the visual servoing model.

Assumption 1: In the uncertain model, the pixel coordinates of the feature points is an estimate value $\hat{\mathbf{p}}_{i}$ related to the true value $\mathbf{p}_{i}$ by the relationship:

$$
\hat{\mathbf{p}}_{i}=\mathbf{p}_{i}+\mathbf{n}
$$

where $\|\mathbf{n}\|_{\infty} \leq \eta$ represents the image noise intensity, $\eta$ is known positive constant.

Assumption 2: An estimate of the intrinsic parameters matrix $\hat{\mathbf{A}}$ is denoted as:

$$
\hat{\mathbf{A}}=\mathbf{A}+\Lambda
$$




$$
\Lambda=\left[\begin{array}{ccc}
\lambda_{1} & 0 & \lambda_{2} \\
0 & \lambda_{3} & \lambda_{4} \\
0 & 0 & 0
\end{array}\right]
$$

where the bounds of $\lambda_{1}, \lambda_{2}, \lambda_{3}, \lambda_{4} \in \mathfrak{R}$ are assumed to be known as:

$\lambda_{i} \in\left[\lambda_{i}^{-}, \lambda_{i}^{+}\right]$

for some limits of $\lambda_{1}^{-}, \lambda_{1}^{+}, \ldots, \lambda_{4}^{-}, \lambda_{4}^{+} \in \mathfrak{R}$

Assumption 3: The depths of the feature points not at infinity. Depending on the distance between the target and the camera, there exist positive constants $z_{i}^{-}$and $z_{i}^{+}$which make the depth within the range of:

$$
\hat{z}_{i} \in\left\lfloor z_{i}^{-}, z_{i}^{+}\right\rfloor
$$

\section{Robust Visual Servoing Controller Design with Efficient TP Model Transformation Method}

For the visual servoing system with the uncertain models (7)-(11), a robust visual servoing control scheme should be considered to minimize the error $\mathbf{e}(t)$ while fulfilling a set of constraints such as visibility, workspace and actuator limitations for all possible uncertainties.

\subsection{The Quasi-Min-Max MPC Formulation for IBVS System}

Considering the spatial velocity of the camera $\mathbf{v}_{c}=\left[v_{c}, \omega_{c}\right] \in \mathfrak{R}^{6}$, which is composed of the linear velocity $v_{c}=\left(v_{x}, v_{y}, v_{z}\right)^{T} \in \mathfrak{R}^{3}$ of the origin of the camera frame and the angular velocity $\omega=\left(\omega_{x}, \omega_{y}, \omega_{z}\right)^{T} \in \mathfrak{R}^{3}$ of the camera frame. Taking pixel coordinates of the feature point as image features $\mathbf{s}_{i}=\left(u_{i}, v_{i}\right) \in \mathfrak{R}^{2}$, the relationship between the time variation of image features and the camera velocity is:

$\dot{\mathbf{s}}_{i}=J_{s_{i}} \mathbf{v}_{c}$

with $\forall i=1,2,3,4$. The image Jacobian matrix, also called interaction matrix $J_{s_{i}} \in \mathfrak{R}^{2 \times 6}$ is: 


$$
J_{s i}=\left[\begin{array}{cccccc}
-\frac{k_{x}}{z_{i}} & 0 & \frac{u_{i}-u_{0}}{z_{i}} & \frac{\left(u_{i}-u_{0}\right)\left(v_{i}-v_{0}\right)}{k_{y}} & -\left(k_{x}+\frac{\left(u_{i}-u_{0}\right)^{2}}{k_{x}}\right) & \frac{k_{x}\left(v_{i}-v_{0}\right)}{k_{y}} \\
0 & -\frac{k_{y}}{z_{i}} & \frac{v_{i}-v_{0}}{z_{i}} & k_{y}+\frac{\left(v_{i}-v_{0}\right)^{2}}{k_{y}} & -\frac{\left(u_{i}-u_{0}\right)\left(v_{i}-v_{0}\right)}{k_{x}} & -\frac{k_{y}\left(u_{i}-u_{0}\right)}{k_{x}}
\end{array}\right]
$$

To use quasi-min-max MPC to control the robotic visual servoing system, the discrete time model is used instead of the continuous time model. The overall system dynamics can be expressed as follows:

$\mathbf{e}_{i}(k+1)=\mathbf{e}_{i}(k)+T_{s} J_{s i}(k) \mathbf{v}_{c}(k)$

where $\mathbf{e}_{i}(k)=\mathbf{s}_{i}(k)-\mathbf{s}^{*}$ is both the states and outputs of the system, $T_{s}$ is the sampling time. The control objective is to tackle the robot position problem in the presence of the system constraints, while the robot and camera models with parametric uncertainties. Quasi-min-max MPC is an effective method to find the optimal control input of system at each sampling time $k$, by solving the constrained infinite-time convex optimization problem, which can be expressed as the following LMI-based minimization problem with input and output constraints [14]:

$\min _{\mathbf{v}_{c}(k \mid k), Q, Y, \gamma} \gamma$

subject to:

$$
\begin{aligned}
& {\left[\begin{array}{cccc}
1 & * & * & * \\
\mathbf{e}_{i}(k \mid k)+T_{s} J_{s i}(k) \mathbf{v}_{c}(k \mid k) & Q & * & * \\
Q_{w}^{0.5} \mathbf{e}_{i}(k \mid k) & 0 & \gamma I & * \\
R_{w}^{0.5} \mathbf{v}_{c}(k \mid k) & 0 & 0 & \gamma I
\end{array}\right] \geq 0} \\
& {\left[\begin{array}{cccc}
Q & * & * & * \\
Q+T_{s} J_{s r} Y & Q & * & * \\
Q_{w}^{0.5} Q & 0 & \gamma I & * \\
R_{w}^{0.5} Y & 0 & 0 & \gamma I
\end{array}\right] \geq 0} \\
& \left|\mathrm{v}_{c l}(k \mid k)\right| \leq \mathrm{v}_{c l, \max } \\
& {\left[\begin{array}{cc}
\mathrm{v}_{c \max }^{2} & * \\
Y^{T} & Q
\end{array}\right] \geq 0} \\
& \left\|\mathbf{e}_{i}(k \mid k)+J_{s i}(k) \mathrm{v}_{c}(k \mid k)\right\|_{2} \leq e_{\max }
\end{aligned}
$$


$\left[\begin{array}{cc}Q & * \\ Q+J_{s r} Y & e_{\max }^{2}\end{array}\right] \geq 0$

where the symbol * induces a symmetric structure of linear matrix inequality. $Q_{w}>0$ and $R_{w}>0$ are two positive definite weighting matrix. $Q$ is the symmetric positive definite matrix and $i=1,2,3,4$ denotes the four image feature point, and $l=1,2, \ldots, 6$ denotes the dimension of current input vector $\mathbf{v}_{c}(k \mid k)$, The current optimal control signal for IBVS is $\mathbf{v}_{c}(k \mid k)$. The future feedback control signal is calculated as $\mathbf{v}_{c i}(k+j \mid k)=F(k) \mathbf{e}_{i}(k+j \mid k), j \geq 1$ and $F(k)=Y Q^{-1} . v_{c l \text {, max }}, s_{\max }$ represent the robot physical limitations and upper limit of the image feature values, respectively. It should be notice that, $J_{s r}$, $\forall r=1, \ldots, R$ represents the convex vertexes of the image Jacobian matrix (13) considering all the uncertainties listed above. Thus, the feasible solution of LMIs (15)-(21) gives an optimal control input that can fulfill the visibility and actuator constraints as well as the possible robot and camera uncertainties.

Obviously, the computational speed of the LMI-based controller mainly depends on the number of convex vertexes of the image Jacobian matrix $J_{s r}, \forall r=1, \ldots, R$. Especially in the presence of uncertain parameters, the increase of the variable parameter dimension in the image Jacobian matrix will also affect the computational complexity. Therefore, it is very important to find an effective method to obtain the proper vertex matrices while reducing the dimension and complexity.

\subsection{Efficient TP Model Transformation for Image Jacobian Matrix}

Similar to Eq.(1), the image Jacobian matrix (13) contains a set of time varying image measurements $\hat{u}_{i}(k), \hat{v}_{i}(k), \hat{z}_{i}(k)$, and the estimated camera intrinsic parameters $\hat{k}_{x}, \hat{k}_{y}, \hat{u}_{0}, \hat{v}_{0}$ with bounded uncertainties. Combine $\hat{u}_{0}$ into $\hat{u}_{i}(k)$, and $\hat{v}_{0}$ into $\hat{v}_{i}(k)$, then the image Jacobian matrix nonlinearly depends on five parameters $p_{i}(k)=\left\{u_{i}(k), v_{i}(k), 1 / z_{i}(k), k_{x}, k_{y}\right\}$. TP model transformation is a very effective method to transform the image Jacobian matrix into polytopic form $J_{s r}$. However, because of the uncertain parameters, an excessive number of TP vertices are extracted, which impose great difficulties on the online calculation of linear matrix inequalities of (15) - (21). Hence, an efficient modified TP Model Transformation method based on the uniform design is implemented to drastically reduce the number of the vertex for the image Jacobian matrix. The procedure can be performed as follows: 
STEP1: Under a certain degree of uniformity measure index, according to the good grid point method, the power grid method and some uniform design method is adopted to get a uniform design (UD) table [27]. Since $p_{i}(k)=\left\{u_{i}(k), v_{i}(k), 1 / z_{i}(k), k_{x}, k_{y}\right\}$ is a 5 -dimensional variable parameter vector, if the number of the grid lines in each dimension is $M$, the uniform design to be obtained is $U_{M}\left(M^{5}\right)$.

STEP2: Discrete the image Jacobian matrix according to the UD method. Define the transformation space $\Omega$ as:

$$
p_{i}(k) \in \Omega:\left[u_{i m}, u_{i M}\right] \times\left[v_{i m}, v_{i M}\right] \times\left[1 / z_{i m}, 1 / z_{i M}\right] \times\left[k_{x m}, k_{x M}\right] \times\left\lfloor k_{y m}, k_{y M}\right\rfloor
$$

in which, $u_{i m}, u_{i M}, v_{i m}, v_{i M}$ are the minimum and maximum ranges of the image point coordinates considering the image measurement errors and the camera calibration errors, $z_{i m}, z_{i M}$ are the minimum and maximum depths between the object and the camera, and $k_{x m}, k_{x M}, k_{y m}, k_{y M}$ are the bounded uncertain ranges of the magnification factor of $x$ and $y$ axis respectively. Therefore, it is necessary to map the level of each factor in the UD table to the closed variable $\Omega$ of the variables, so that to get the discrete point $g_{i}=\left(g_{1, i}, \ldots g_{5, i}\right), i=1,2 \ldots M$. The image Jacobian matrix $J_{s i}(p(k))$ is discretized by sampling over the grid points, and the result is stored into the tensor $\mathbf{J}_{s i}^{D} \in \mathfrak{R}^{M \times 2 \times 6}$.

STEP3: HOSVD is applied to the first dimension of tensor $\mathbf{J}_{s i}^{D}$. Discard all zero or a smaller singular value $\sigma_{k}$ and the corresponding singular value vector, the following relation holds:

$\mathbf{J}_{\mathbf{s i}}^{\mathbf{D}} \approx \mathbf{J}_{s i} \times_{1} U_{1}$

where $\quad \mathbf{J}_{s i} \in \mathfrak{R}^{T \times 2 \times 6}$ is the system core tensor obtained after transformation, $T \leq M . U_{1} \in \mathfrak{R}^{M \times T}$ is the matrix of weight coefficients corresponding to the core tensor. $\varepsilon$ represents the upper bound of the approximate error in the above transformation process. Further transformations like SN (Sum Normalization), NN (Non-Negative), and NO (Normality) or INO-RNO (InverseNO and Relaxed-NO) could be executed in order to get the better application effect. Thus, the convex vertexes of the image Jacobian matrix $J_{s r}, \forall r=1, \ldots, R$ are obtained, with the vertex number of $R=T$, which meet the requirement of LMIs (17) and (20) to get the optimal control input of the visual servoing system. 


\section{Simulation and Experiment}

\subsection{Simulation Results}

The simulations are carried out using MATLAB 7.1, on PC Pentium CPU G2020 $2.9 \mathrm{GHz}$ in Microsoft Windows 7 operating system. The image Jacobian matrix nonlinearly depends on five parameters. The transformation space $\Omega$ is [-176176;-132132;0.720;350500;350500]. If we apply the classical TP model transformation method as in the reference [14], $3 \times 3 \times 2 \times 3 \times 3=162$ vertex of the image Jacobian matrices is obtained. Owing to the large number of the vertex matrices, real-time is impossible to achieve during the application of the visual servoing system.

The efficient TP model transformation method based on uniform design can be applied to relax the complexity issues. Define the discretization grids based on the uniform design $U_{200}\left(200^{5}\right)$ which $C D_{2} \leq 0.0207$. Then, obtain the discrete tensor $\mathbf{J}_{s i}^{D} \in \mathfrak{R}^{200 \times 2 \times 6}$. Execute HOSVD and discard all zero singular values, the corresponding weighting coefficient functions shown in Figure 1 and the resulting number of vertex image Jacobian matrices is the same as the parameter number, namely $J_{s r}, \forall r=1, \ldots, 5$.

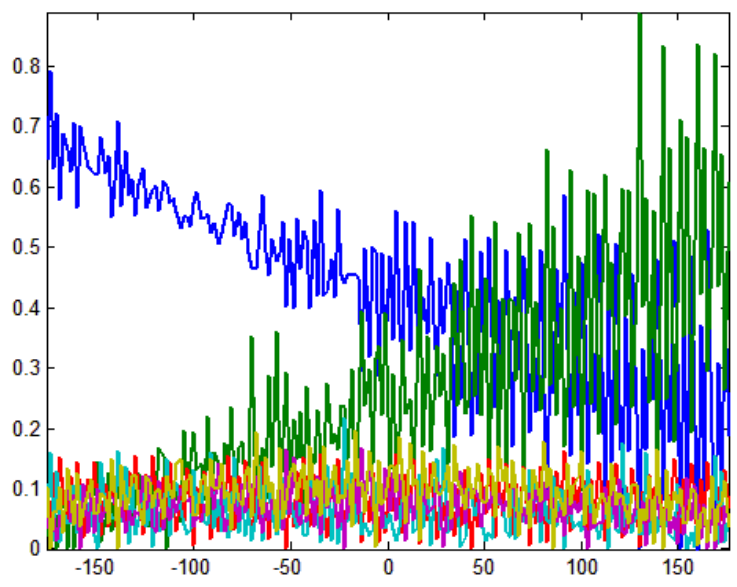

Figure 1

The discretized weighting functions

To achieve a visual servoing task, a large displacement in the depth direction from the initial pose to the desired pose are considered, which is the same as reference [14], and the initial and the desired poses of the camera are listed in Table 1. Assuming that the camera is coarsely calibrated, the estimated values of the camera intrinsic parameters are the maximum uncertain boundary values. The true camera intrinsic matrix and its estimate values are: 


$$
\mathbf{A}=\left[\begin{array}{ccc}
418 & 0 & 160 \\
0 & 418 & 120 \\
0 & 0 & 1
\end{array}\right], \hat{\mathbf{A}}=\left[\begin{array}{ccc}
500 & 0 & 192 \\
0 & 350 & 144 \\
0 & 0 & 1
\end{array}\right]
$$

Table1

Initial and desired poses of simulation task

\begin{tabular}{ccccccc}
\hline \hline Pose & $X / \mathrm{m}$ & $Y / \mathrm{m}$ & $Z / \mathrm{m}$ & $R / \mathrm{rad}$ & $P / \mathrm{rad}$ & $Y / \mathrm{rad}$ \\
\hline $\begin{array}{c}\text { Initial Pose } \\
\text { Desired } \\
\text { Pose }\end{array}$ & -0.022 & 0.004 & 0.584 & -0.349 & 2.793 & -3.143 \\
\hline \hline
\end{tabular}

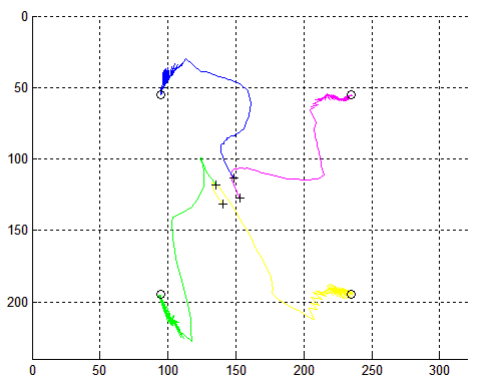

a) Image plane
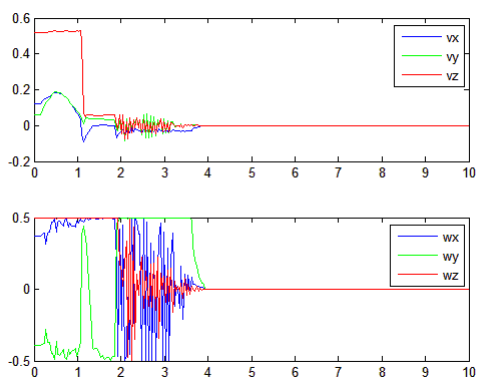

c) Camera Cartesian velocity

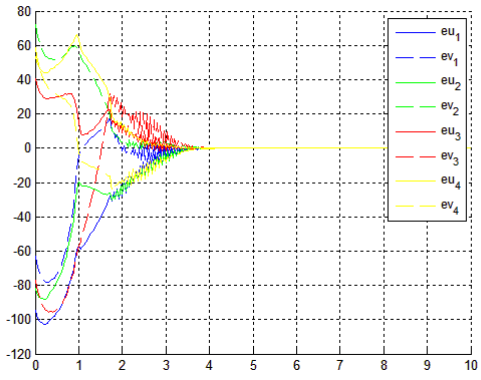

b) Image errors

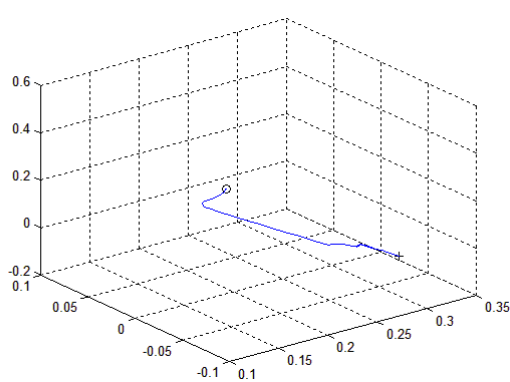

d) Camera 3D trajectory

Figure 2

Simulation results for the proposed method

Depth $1 / z_{i}=16$ is a selected fixed value between the object and the camera. Moreover, the image measurements are added random noises in 5 pixels with uniform distribution. Yalmip toolbox is adopted to solve optimization involving the LMIs. The simulation results of both the proposed algorithm and reference [14] ([14] without considering the model uncertainties) are given, as shown in Figure 2 and Figure 3, respectively. 


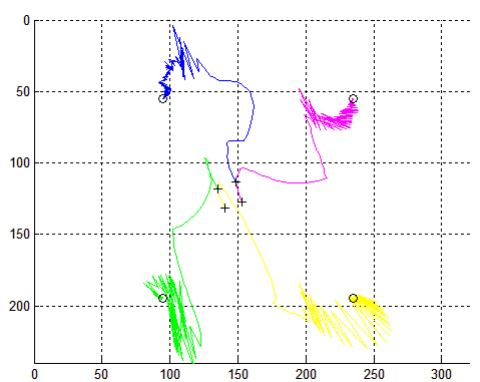

a) Image plane
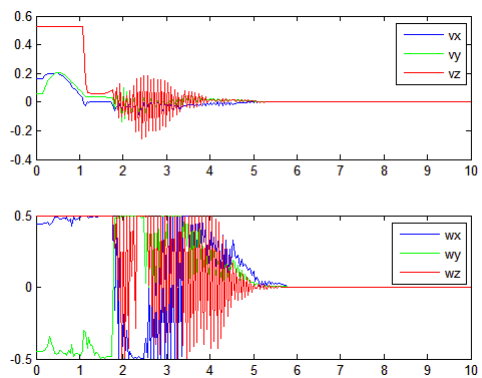

c) Camera Cartesian velocity

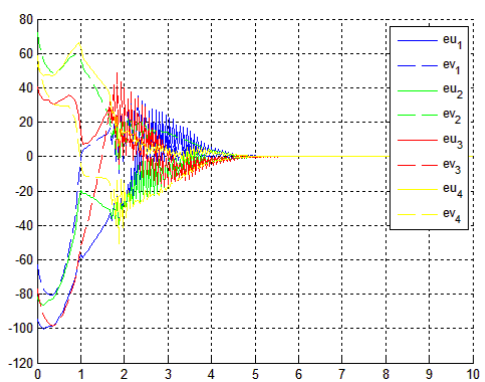

b) Image errors

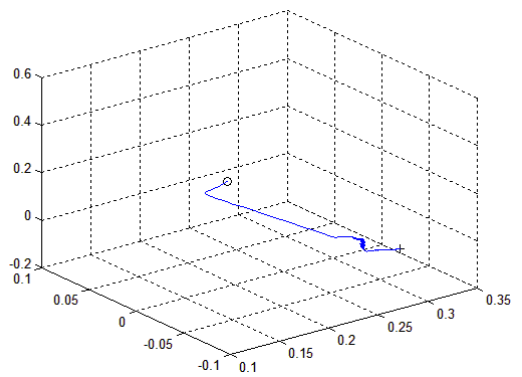

d) Camera 3D trajectory

Figure 3

Simulation results for reference [14]

Due to the system constraints and the disturbances, there are some oscillations near the desired position. However, because of considering the system uncertainties when constructing the TP models of the image Jacobian matrix, the proposed method has an obviously better control performance. What's more, its operation speed met the requirements of online control.

\subsection{Experimental Results}

In this section, a 6-DOF ABB IRB120 manipulator equipped with an eye-in-hand camera, is carried out for the experimental results to verify the propose method. Known that the resolution of the camera is $640 \times 480$, the estimation of its intrinsic parameter matrix is

$$
\hat{A}=\left[\begin{array}{ccc}
960.3855 & 0 & 288.9303 \\
0 & 951.3275 & 234.8129 \\
0 & 0 & 1
\end{array}\right]
$$

Meanwhile, taking image centers of four color circles as feature points, image measurement errors will be introduced. Three visual servoing tasks are listed to verify the effectiveness of the algorithm. 
Task 1 is a normal visual servoing task contains a small range of rotation. The initial pose of 6-DOF robot's joint angle is $q_{0}=[-1.62,0.42,-0.44,0.03,1.71,2.18]$ in radian and the desired target point coordinates is [286,196;413,143;472,272;343,328].

For task 2, the feature points of the object at both the initial pose and the desired pose are very close to the FOV boundary. The initial pose of 6-DOF robot's joint angle is $q_{0}=[-2.21,0.63,-0.66,0.35,1.65,1.65]$ in radian and the desired target point coordinates is $[283,13 ; 412,20 ; 401,148 ; 275,141]$.

And for task 3, a large displacement in the depth direction from the initial pose to the desired pose are considered. The initial pose of 6-DOF robot's joint angle is $q_{0}=[-1.54,0.21,0.33,-0.01,1.01,2.37]$ in radian and the desired target point coordinates is $[259,107 ; 358,141 ; 323,240 ; 224,206]$.

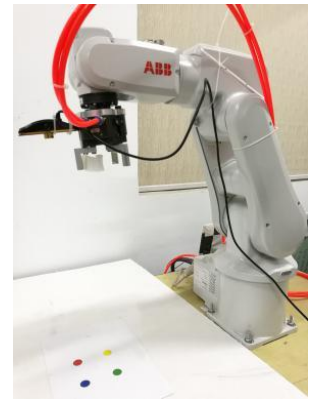

(a) Initial pose

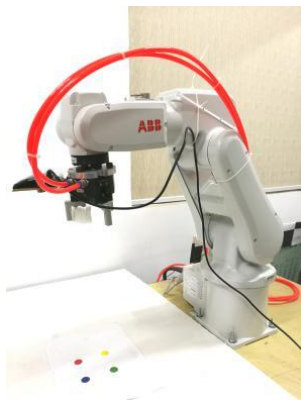

(b) Desired pose

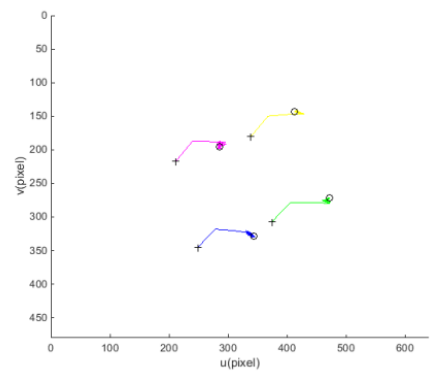

(c) Image plane

Figure 4

Simulation results of Task 1

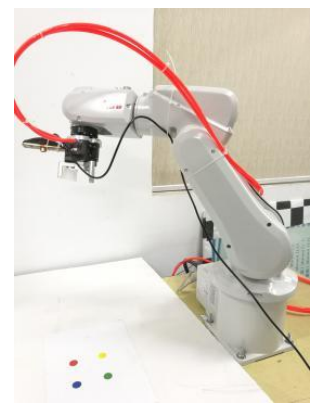

(a) Initial pose

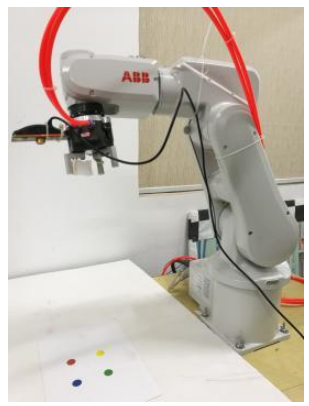

(b) Desired pose

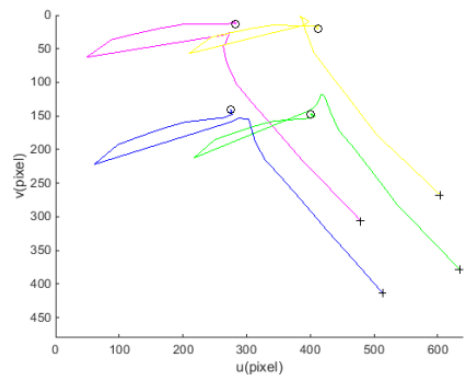

(c) Image plane

Figure 5

Simulation results of Task 2

The experimental results are shown in Figures 4-6. Understand that in the image plane, the cross symbols ("+") represent the initial position of the visual feature points, and the circle symbols ("॰") represent the desired feature points. It can be 
seen that all the visual servoing tasks have been successfully completed online, the proposed algorithm is effective.

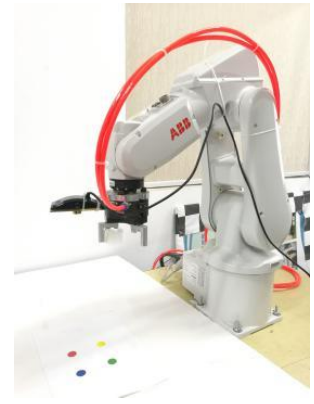

(a) Initial pose

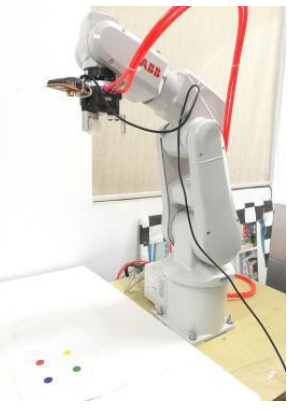

(b)Desire pose

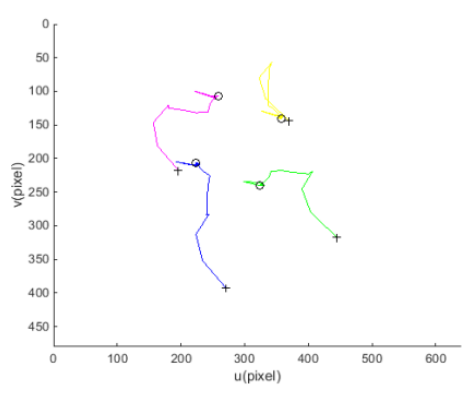

(c) Image plane

Figure 6

Simulation results of Task 3

\section{Conclusion}

This paper proposed a Robust Optimization Visual Servoing control scheme, which depends on an efficient TP model transformation, based on uniform design, which can handle the uncertain system parameters in the image Jacobian matrix. The proposed method obtains the discrete tensor of image Jacobian through uniform design. The result is that the computational load of LMIs, in the quasimin-max MPC controller is greatly reduced. Simulation and experimental results show that the algorithm has superior robustness in model uncertainties and in realtime performance.

\section{Acknowledgement}

Research supported by the Fundamental Research Funds for the Central Universities under Grant B200202220, National Natural Science Foundation of China under Grant 61403122, 61873086 and Changzhou Science and Technology Support \& Social Development Project under Grant CE20195021.

\section{References}

[1] F. Chaumette, S. Hutchinson: Visual servo control, Part I: Basic approaches, IEEE Transaction on Robotics and Automation Magazine, Vol. 13, No. 4, pp. 82-90, 2006

[2] F. Chaumette, S. Hutchinson: Visual servo control, Part II: Advanced approaches, IEEE Transaction on Robotics and Automation Magazine, Vol. 14, No. 1, pp. 109-118,2007

[3] K. S. Hwang, M. H. Chung, W. C. Jiang: Image based visual servoing using proportional controller with compensator, IEEE International Conference on Systems, Man, and Cybernetics, pp. 347-352, 2015 
[4] B. Thuilot, P. Martinet, L. Cordessed, J. Gallice: Position based visual servoing: keeping the object int he field of vision, IEEE International Conference on Robotics and Automation, 2002

[5] E. Mails, F. Chaumette, S. Boudet: 2 1/2 D visual servoing, IEEE Transactions on Robotics and Automation, Vol. 15, No. 2, pp. 238-250, 1999

[6] I. Siradjuddin, L. Behera, T. McGinnity, and S. Coleman: Image-based visual servoing of a 7-DOF robot manipulator using an adaptive distributed fuzzy PD controller, IEEE/ASME Transaction Mechatronics, Vol. 19, No. 2, pp. 512-523, 2014

[7] W. F. Xie, Z. Li, X. W. Tu, C. Perron: Switching control of image-based visual servoing with laser pointed in robotic manufacturing system, IEEE Transaction on Industrial and Electronics, Vol. 56, No. 2, pp. 520-529, 2009

[8] M. Bakthavatchalam, O. Tahri, F. Chaumette: A direct dense visual servoing approach using photometric moments, IEEE Transactions on Robotics, Vol. 34, No. 5, pp. 1226-1239, 2018

[9] F. J. Wang; L. L. Song; Z. Liu; F. Zhang: Adaptive visual servoing control of robot with unknown hysteresis constraint, $35^{\text {th }}$ Chinese Control Conference, pp. 6944-6949, 2016

[10] H. B. Shi, G. Sun, Y. P. Wang, K. S. Hwang: Adaptive image-based visual servoing with temporary loss of the visual servoing, IEEE Transaction on Industrial Informatics, Vol. 15, No. 4, pp. 1956-1965, 2019

[11] T. T. Shen, G. Chesi: Visual servoing path-planning with elliptical projections, Informations in Control, Automation and Robotics, Vol. 430, pp. 30-54, 2017

[12] P. Munoz-Benavent, L. Gracia, J. Solanes, A. Esparza: Robust fulfillment of constraints in robot visual servoing, Control Engineering Practice, Vol. 71, pp. 79-95, 2018

[13] G. Allibert, E. Courtial, and F. Chaumette: Predictive control for constrained image-based visual servoing, IEEE Transactions on Robotics, Vol. 26, No. 5, pp. 933-939, 2010

[14] T. T. Wang, W. F. Xie, G. D. Liu, and Y. M. Zhao: Quasi-min-max model predictive control for image-based visual servoing with tensor product model transformation, Asian Journal of Control, Vol. 17, No. 2, pp. 402416, 2015

[15] A. Hajiloo, M. Keshmiri, W. F. Xie, and T. T. Wang: Robust online model predictive control for a constrained image-based visual servoing, IEEE Transaction on Industrial and Electronics, Vol. 63, No. 4, pp. 2242-2250, 2016 
[16] S. John, J. O. Pedro: Neural network-based adaptive feedback linearization control of antilock braking system, International Journal of Artificial Intelligence, Vol. 10, No. S13, pp. 21-40, 2013

[17] H. Y. Du, J. Yan, Y. H. Fan: A state and input constrained control method for air-breathing hypersonic vehicles, Acta Polytechnica Hungarica, Vol. 15, No. 3, pp. 81-99, 2018

[18] C. Pozna, R. E. Precup: An approach to the design of nonlinear statespace control systems, Studies in Informatics and Control, Vol. 27, No. 1, pp. 5-14, 2018

[19] P. Baranyi, D. Tikk, Y. Yam, and R. J. Patton: From different equations to PDC controller design via numeri cal transformation, Comput. Ind., Vol. 51, No. 3, pp. 281-297, 2003

[20] P. Baranyi: TP model transformation as a way to LMI-based controller design, IEEE Transactions on Industrial and Electronics, Vol. 51, No. 2, pp. 387-400, 2004

[21] P. Baranyi: TP model transformation as a manipulation tool for QLPV analysis and design, Asian Journal of Control, Vol. 17, No. 2, pp. 497-507, 2015

[22] P. Baranyi: Extracting LPV and qLPV structures from state-space functions: a TP model transformation based framework, IEEE Transactions on Fuzzy Systems, DOI 10.1109/TFUZZ.2019.2908770

[23] A. Szollosi, P. Baranyi: Influence of the Tensor Product model representation of qLPV models on the feasibility of Linear Matrix Inequality, Asian Journal of Control, Vol. 18, No. 4, pp. 1328-1342, 2015

[24] G. Zhao, D. Wang, Z. Song: A novel tensor product model transformationbased adaptive variable universe of discourse controller, Journal of the Franklin Institute, Elsevier, Vol. 353, No. 17, 2016

[25] V. C. S. Campos, F. O. Souza, L. A. B. Torres, R. M. Palhares: New stability conditions based on piecewise fuzzy lyapunov functions and tensor product transformations, IEEE Transactions on Fuzzy Systems, Vol. 21, No. 4, pp. 760-784, 2013

[26] P. Kornodi: Tensor product model transformation-based sliding surface design, Acta Polytechnica Hungarica, Vol. 3, No. 4, pp. 23-25, 2006

[27] J. F. Cui, K. Zhang, T. H. Ma: An efficient algorithm for the tensor product model transformation. International Journal of Control, Automation and Systems, Vol. 14, No. 5, pp. 1205-1212, 2016

[28] J. F. Cui, K. Zhang, M. B. Lv: Tensor product distributed compensation control method based on uniform design, Control and Decision, Vol. 30, No. 4, pp. 745-750, 2015 